\title{
New ways with aggregation
}

A new treatment of certain problems of aggregation, neat in itself, promises to be a helpful adjunct to established (but imprecise) methods.

SiMPLE-MINDED problems usually conceal a catch somewhere. Here is one, taken from Physical Review Letters for 11 June. Suppose there is a gun that fires bullets in the same direction at regular intervals. To simplify (and to make the problem onedimensional), suppose there is no such thing as gravity. If all the bullets left the muzzle with the same velocity, there would be no problem, but merely a train of bullets following each other at regular intervals in the direction of the axis of the barrel. In that case, there is nothing much to say.

The fun begins when you suppose that the muzzle velocities are not all the same. Then, some bullets will overtake those ahead of them and may then stick together. Given enough variability of muzzle velocity, substantial aggregations of bullets, all travelling in the same direction, may be formed. G. F. Carnevale and W. R. Young, from the Scripps Institution of Oceanography in La Jolla, California, and Y. Pomeau, from the Ecole Normale Supérieure in Paris, call it "ballistic agglomeration" (Phys. Rev. Lett. 64, 2913; 1990). The surprise is that nobody seems to have thought of tackling it before this. The authors' acknowledgement of support from the US Office of Naval Research should not be taken too literally; even in naval battles, shells do not often collide with each other, but vorticies and convection plumes do so routinely.

What are the simple expectations of the problem? Both mass and momentum will be conserved at every collision. The velocities of individual projectiles can be taken to be randomly distributed in a finite range about some mean value. What can be said about the long-term behaviour of such a system? Some may say nothing. Carnevale et al. suggest that others, more sophisticated, and brought up on textbooks of fluid mechanics, will argue that the pattern of agglomeration a long way from the muzzle will depend on the mass flux (which is constant and equal to $m_{0} / \tau_{0}$, where $m_{\mathrm{t}}$ is the mass of each projectile and $\tau_{0}$ the interval between firings), the momentum flux (also constant and equal to $m_{0} u / \tau_{0}$ where $u$ is the mean velocity) and the distance from the muzzle $(x)$.

Distance, here, is a proxy for time, and it would be reasonable to ask for some answer to the question of how the masses of the agglomerations reaching $x$ are distributed; some will consist of single bullets, others of several. Textbook writers have a ready solution. Since there are three variables (two fluxes and a distance), there is only one way of making a function which has dimensions of mass and which is itself a function of $x$.

This simple argument leads to the conclusion that the distribution of mass is the function $F_{p}^{2} x / F_{m}$, where $F_{p}$ and $F_{m}$ are the fluxes of momentum and of mass respectively. The immediate thing to say about this result is that it is incorrect. The authors use a numerical simulation to show that even when a train of 100,000 projectiles has been launched, with velocities taken randomly from an interval 50 per cent above and below the mean, there are fewer than 1,000 agglomerations in being, but that many of these consist of only a single projectile. The surprise is that there are so many singles left.

Evidently this is an over-simple version of what must be the real problem - that of telling what happens to aggregating particles which are free to move in several dimensions. The authors rightly say that aggregation by coalescence at every collision is a very special case of a more complicated phenomenon. The surprise, again, is that while some things can be said about the general problem, only the onedimensional case has been dealt with. But the argument is memorably neat.

To visualize what happens at agglomeration, the authors assume that every aggregation of unit particles assumes the configuration of a sphere. The problem is to find the mass distribution as a function of time, $t$. Because of the adjustment to a sphere, $m(t)=r^{\prime}(t)$, where $r(t)$ is the radial distribution and $D$ the dimensionality. At the very outset of the agglomeration, the interval between collisions will be the ratio of the volume of each particle to the volume swept out in unit time specifically, $a_{0}^{d} / u_{0} r_{00}{ }^{b-t}$ where $a$ stands for volume and the zeros emphasize that this is right at the beginning.

Now what happens as time goes on, say after the passage of, say, $q$ units of the characteristic time $\tau_{0}$ ? Evidently some unit spheres will have agglomerated into larger structures; indeed, the expected number of units in each agglomerate will be some function $N(q)$. But the problem is then the same as at the beginning except that the particles are larger. This already says some uscful things - the velocity of the aggregations declines inversely as the square root of the function $N(q)$, for example.

Now comes the trick. The authors choose a second later time, and regard that at $q \tau_{0}$ as the starting point, which leads to a similar set of scaling rules, which must be identical in form with the first. The end result is that $N(q)=q^{2(D)(D)+2)}$ (where $D$ is dimensionality). In short, in one dimension, particles grow as $q^{2 / 3}$, in three dimensions as $q^{\text {w.5. }}$. There are numerical solutions (in one dimension) to prove the point.

Two interesting issues arise, of which the most obvious is why so much hangs on simulation in one dimension. The explanation is humdrum - the algorithms are tractable only if the particles are very small.

The more interesting issue is what might be expected to come out of studies such as these. The authors list a whole series of problems to which their work may be relevant, ranging from the condensation of stellar nebulae into planets to the aggregation of colloids and the interaction of hydrodynamic structures. Although all these are problems which can be (and have been) tackled differently, a new way of working may prove especially valuable.

So much is nicely illustrated by the bestknown model of aggregation, that of "diffusion-limited aggregation" originally due to Edward Witten at Santa Barbara, California. There the guiding idea is that particles diffuse towards a growing aggregate by random walks from a remote periphery, and stick whenever they reach a site (on a normally two-dimensional lattice) adjacent to another already occupied. Unvarnished, this process builds lacy structures whose dimensions prove to be fractional - they are fractal structures, yet many hours of elaborate central processor time spent on the numerical simulation of such models have not solved many practical problems - but there are some intriguing applications in the scattering of light by smoke particles. It is much more important that this model has lent itself to the prediction of some general scaling laws, and to a kind of intuitive sense of the physics of the problem.

Much the same will probably happen with its new model. Apart from its problem of planetary aggregation (which is essentially three-dimensional) there are few obvious applications likely to be of direct utility. Plainly, one should not expect more. Despite the effort and the time that goes into the development of these models, their lasting value is probably hcuristic - people have a better insight than previously.

John Maddox 